\title{
Der Einfluß der Elbe auf die Verunreinigung der Nordsee ${ }^{1}$
}

\author{
Hubert Caspers \\ Hydrobiologische Abteilung des Zoologischen Staatsinstituts tund Museums, \\ Hamburg
}

\begin{abstract}
Influence of the Elbe on the pollution of the North Sea. The inflow of organic rest material from rivers into the sea represents a kind of pollution resulting in an increased trophic level. Dilution in the river as well as the general geographic and hydrographic situations determine the marine equilibrium resulting from the supply of nutrients. In the Elbe estuary exist all kinds of transitions between mixo-oligohaline and mixo-polyhaline zones; the location of which may shift over decades or, in part, over short periods of time. As a consequence, the benthos is exposed to extreme variations. Due to the alternation of tides, the plankton remains longer within the individual salinity zones than would be the case in a one directional flow system. Hence typical planktonic biocoenoses can develop in regional successions. In this regard the phytoplankton with its fast reproduction rates is especially favoured. Due to hydrographical reasons a turbidity zone is formed in the oligoto merohaline areas containing primarily remains of the limnic production. An estuary system is, by its nature, adjusted to accept increased decomposition loads; addition of organic anthropogenic remains is, therefore, tolerable without significant biocoenotic consequences. The inflow of dissolved nutrients from the Elbe results in an increase of the trophic niveau and a "secondary pollution" in the outer regions of the estuary. Mud flats and deeper sea areas are capable of accepting large amounts of detritus. The question, to what extent these areas may be capable of tolerating additional organic waste products without negative biological consequences, is discussed.
\end{abstract}

\section{EINLEITUNG}

Der Einstrom von Süßwasser in das Meer muß zwangsläufig zum Absterben großer Mengen abdriftender Süßwasserorganismen führen mit der Folge einer Trophiesteigerung der Küstenbereiche. Biologisch gesehen ist also hierdurch eine „Verschmutzung" gegeben, indem allochthones organisches Restmaterial einem „Vorfluter" eingebracht wird. Hierbei ist zu unterscheiden zwischen dem noch partikulären, also erst in der Dekomposition begriffenen Detritus und den bereits im Flußablauf mineralisierten Substanzen, die direkt dem Nährstoffspiegel des Vorfluters - also dem marinen Küstenbereich - zukommen. Der Detritus wird weiter in das offene Meer verdriftet bzw. in Küstennähe sedimentiert. Die unterschiedliche Dynamik dieser Abbauprozesse be-

\footnotetext{
1 Mit Unterstützung der Deutschen Forschungsgemeinschaft.
} 
stimmt zusammen mit dem vorgegebenen Nährstoffniveau des Meeres die aktuelle Primärproduktion und damit den Trophiegrad (zur Relation Trophie-Dekomposition vgl. Caspers \& Karbe 1967).

\section{ERGEBNISSE UND DISKUSSION}

Unter "Verschmutzung" des Meeres - dem Generalthema dieses internationalen Symposions der Biologischen Anstalt Helgoland - wird üblicherweise die schädigende anthropogene Anderung eines natürlich vorhandenen trophischen Equilibriums durch Einleitung von häuslichen und industriellen Abwässern verstanden. Grundsätzlich sollten wir aber den Zustrom von organischem Restmaterial durch die Flïsse, sofern hierdurch eine Verschiebung des biologischen Gleichgewichts eintritt, ebenfalls in den Komplex einer "Verschmutzung" mit einbeziehen. Mit diesem Hinweis ist bereits ausgedrückt, daß nicht jede Steigerung des Trophiespiegels auch eine Schädigung der vorhandenen Biocoenose darzustellen braucht, sondern daß sogar eine begrenzte Zufuhr von Nährstoffen eine Steigerung der Produktion zur Folge haben kann, die nicht als negativ zu werten ist. Dies triff in gleichem Umfang für eine "natürliche" wie für eine anthropogene Nährstoff-Einleitung $z u$.

Entscheidend für den Grenzwert, bei dem eine negative Beeinflussung des Equilibriums - also eine "Verschmutzung" - stattfindet, ist die Relation der eingebrachten Menge zur Rate ihrer Verdünnung im Vorfluter. Hinzu kommt jedoch das vorgegebene - regional und jahreszeitlich unterschiedliche - trophische Niveau des Auffanggebietes. Die Kapazität, allochthones, einer Dekomposition zugängliches Material in ein vorhandenes Nährstoffniveau aufzunehmen, ist abhängig vom Zustand und der Aktivität der Biocoenose; ein höher trophisch gekennzeichnetes Meeresgebiet hat zunächst eine größere Aufnahmefähigkeit für zugeführte organische Stoffe und Nährsalze als ein auf niedrigem Gesamt-Nährstoffniveau eingependeltes System. Die Zuleitung von Giften (in weitestem Sinne) führt andererseits in allen trophischen Niveaustufen zu Schädigungen, deren Ausmaß nicht nur von dem Verdünnungsgrad, sondern auch von der Reaktion des einzelnen Organismus auf die spezifische Giftwirkung abhängt. Als Effekt ergibt sich dann eine Behinderung des gesamt-biocoenotischen Gefüges.

Die Auswirkung organischer und anorganischer Einleitungen ist im Küstenbereich stark von der geographischen Situation bestimmt. Für die Flußmündungen ist entscheidend, ob das abfließende Süßwasser zunächst in Buchten oder Lagunen eine langsame Vermischung mit dem Meerwasser erfährt oder ob der Ausstrom in ein offenes und eventuell durch starke Querströmung geprägtes Meeresgebiet geht; auch der Küstenabfall und die Tiefe des marinen Vorgebietes wirken sich maßgebend aus.

Noch spezieller sind die Verhältnisse in Aestuaren, also in offenen Flußmündungen, die durch den marinen Gezeitenstrom beeinflußt sind mit der Folge einer Vermischung des "Oberwassers" mit dem Salzwasser in allen Abstufungen vom mixooligo- bis zum -polyhalinen Bereich. Die kontinuierliche Zonierung der Halinität bedeutet im Hinblick auf die biocoenotische Entwicklung und die Selbstreinigungskraft des aestuarinen Vorfluters ein Spektrum unterschiedlicher Potenz. Diese Zusammenhänge sollen im folgenden genauer geschildert werden, da sie für die Frage nach dem 
Einfluß eines Aestuars auf den "Vorfluter Meer" von entscheidender Bedeutung sind. Es ergibe sich in der vorliegenden. Zusammenstellung allerdings die Notwendigkeit, diese Analyse auf das Elbe-Aestuar zu beschränken; eine vergleichende Untersuchung der differierenden Verhältnisse anderer Flußmündungen der Nordsee - hierbei wäre vor allem auch die Themse heranzuziehen - und Aestuaren in verschiedenen Klimabereichen muß einer speziellen Arbeit vorbehalten bleiben. Die Elbe kann aber als eine Art Prototyp für die Demonstration solcher aestuarinen Zusammenhänge gelten.

Die Elbe-Mündung kann beanspruchen, der bisher am eingehendsten untersuchte Aestuar-Biotop zu sein. Bereits um 1860 wurden hier biologische Beobachtungen gemacht (Kirchenpauer 1862); DaHL (1892) veröffentlichte den ersten Versuch einer umfassenden Analyse, die dann von Vouk und seinen Mitarbeitern erweitert wurde (Hamburgische Elb-Untersuchung 1903-1909). Diese Untersuchungen fanden in der „Hydrobiologischen Abteilung“ in Hamburg einen Mittelpunkt, seitdem diese zunächst überhaupt als „Abteilung für Elbuntersuchungen“ gegründet worden war (vgl. CASPERS 1961). HeNTSCHEL, seit 1919 Leiter dieser Abteilung, berichtete bei der Gründungsversammlung der Internationalen Vereinigung für theoretische und angewandte Limnologie in Kiel (Hentschel 1923) über die Eigenart dieses Lebensraumes und ließ in den folgenden Jahren durch seine Schüler spezielle Untersuchungen im Aestuar machen. Diese Arbeiten wurden nach dem Tode HeNTSCHels 1945 wieder aufgegriffen mit dem Ziel einer umfassenden Studie des Gesamt-Lebensraumes (vgl. Caspers 1949-1959). Aus diesen in der Hydrobiologischen Abteilung entstandenen Untersuchungen sollen im folgenden die Komplexe herausgegriffen werden, die für die Frage nach der Beeinflussung der südlichen Nordsee durch die Elbe von Bedeutung sind.

Wie Abbildung 1 zeigt, haben wir im Elbe-Aestuar zwischen den Watten der "Außenelbe“ im großen und ganzen einen mixo-polyhalinen bis $\alpha$-mesohalinen Vermischungsgrad (Nomenklatur vgl. "Venice System", CASPERS 1959 a), während im eigentlichen Aestuar-Schlauch dann kontinuierlich der Übergang bis zur oberen Grenze des oligohalinen Brackwassers (etwa bei Glïckstadt) zu verfolgen ist. Allerdings ist die Ausdehnung der einzelnen Halinitätsstufen (als festgelegte Bereiche im Kontinuum des Halinitätsspektrums) starken jahreszeitlichen und zum Teil kurzfristigen Verschiebungen ausgesetzt, welche die sowieso durch die Tidenrhythmik gegebene Verschiebung im Auf- und Abpendeln der Wasserkörper überlagern (vgl. auch KüHL \& MANN 1953, Bursche, KüHL \& MANn 1958, KüHL 1966). Wesentlich sind die Menge des zugeführten "Oberwassers" und der jeweilige Windstau. Oberhalb der mixo-oligohalinen Zone ist durch den Gezeitenstau der Tideströmung noch eine relativ lange Strecke im Süßwasserbereich $z u$ verfolgen bis zur oberen Flutgrenze (etwa $50 \mathrm{~km}$ oberhalb Hamburgs), die als eigentliche Grenze des Aestuars aufzufassen ist (vgl. КотнÉ 1961, Lucht 1964, Grimm 1968).

Die starken hydrographischen Schwankungen beschränken die Ansiedlung der Benthos-Organismen auf Arten, die den starken astatischen Verhältnissen angepaßt sind. Für die im Wasser driftenden Organismen - vornehmlich also die Plankter - bedeutet das Auf- und Abpendeln der Wasserkörper eine Verlängerung der Verweilzeit in Bereichen einer bestimmten Halinität. Hierdurch ergibt sich als entscheidendes Biotop-Kriterium, daß die Aestuar-Strecke als eine Folge von relativ statischen Wasserkörpern aufzufassen ist, in denen jeweils eine Kette planktischer Lebensgemeinschaften 
ihren vollen Entwicklungszyklus durchlaufen können, d. h. der Abdrift in letale Halinitätsbereiche durch die Produktionsintensität begegnet wird. So existieren typische Plankton-Biocoenosen: im Süßwasserbereich die Melosira-Brachionus-Gesellschaft, oberhalb der Brackwassergrenze die Actinocyclus normanni-Eurytemora affinis-Gesellschaft, in der mixo-oligohalinen Zone die Synchaeta bicornis-, in der mesohalinen Zone die Coscinodiscus commutatus- und in der polyhalinen Zone eine vornehmlich marine Planktongesellschaft (vgl. ScHulz 1961).

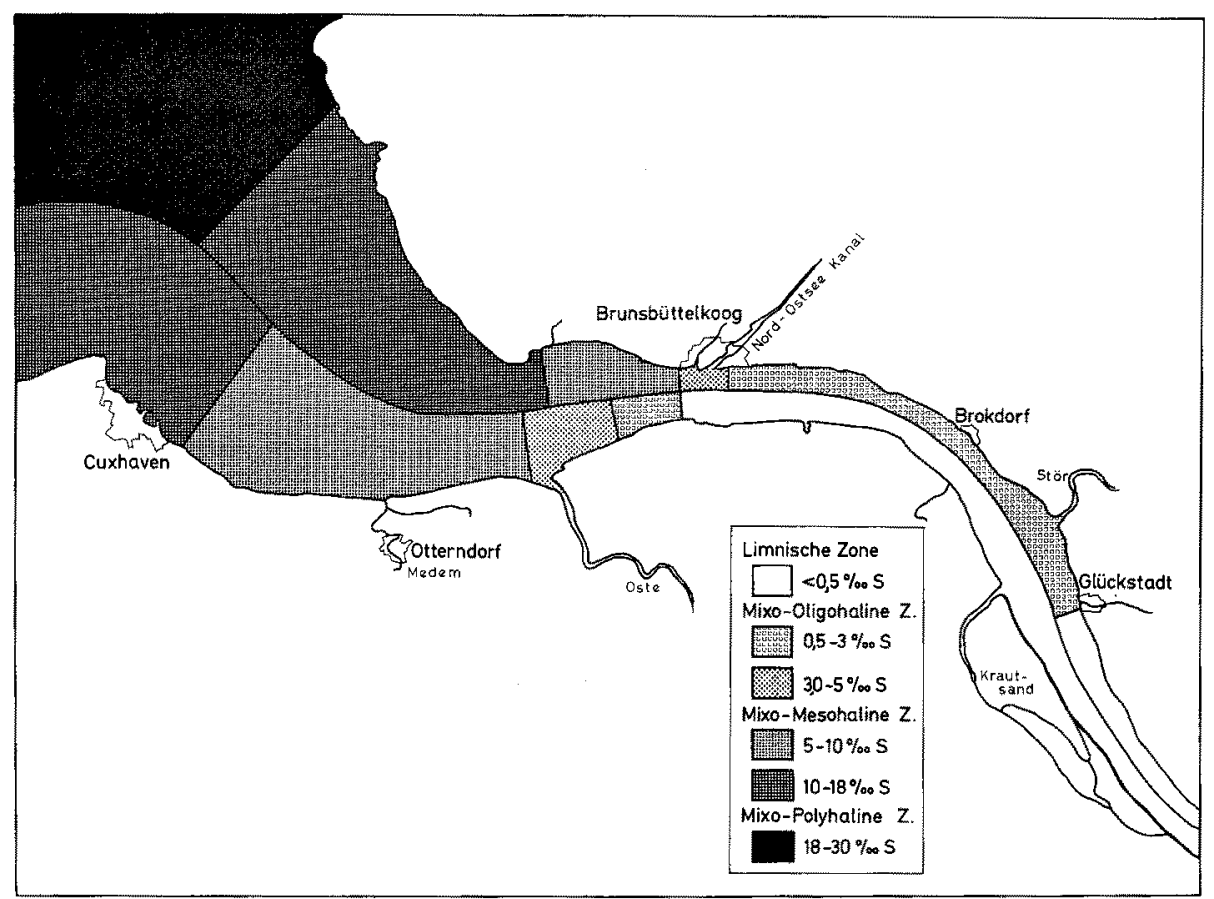

Abb. 1: Mögliche Verschiebung der Brackwasser-Regionen im Elbe-Aestuar. (Nach CAspers 1959a, umgezeichnet)

Giere (1968) gibt in bezug auf das Vorkommen der Zooplankter folgende Zonierung an:

(A) Externer Aestuarbereich. Region 1: Eumarines Plankton (FS „Elbe I" [31\%] bis FS „Elbe II“ [25\% $\%$ S]). Region 2: Euryhalines Meroplankton (oberhalb FS „Elbe II“ [24\%] bis Cuxhaven-Kugelbake [18\% \% ]).

(B) In terner Astu a rbere ich. Region 3: Marin-brackiges Mischplankton (Cuxhaven [ca. 17\%] bis Otterndorf [10\% S]). Region 4: Limnisch-brackiges Mischplankton ( $\beta$-meso- und oligohaliner Bereich bis zur oberen Brackwassergrenze).

Trotz der verlängerten Verweilzeiten von Planktern mit der dadurch gegebenen Stabilisierung einer Kette von planktischen Lebensgemeinschaften bedeutet die Abdrift für die Individuen dieser Populationen den Ubergang in Halinitätszonen, an die sie physiologisch nicht angepaßt sind, so daß ständig in Relation zur Produktionsgröße 
erhebliche Mengen an lebender organischer Substanz zum Absterben kommen. Vor allem ist es die Grenze zwischen dem limnischen und dem oligohalinen Bereich, die für die meisten Süßwasserplankter eine Existenzbarriere darstellt (Abb. 2). Diese Zusammenhänge werden zur Zeit durch meinen Mitarbeiter Dipl.-Biol. I. Nöt'HLIch bearbeitet (vgl. auch NörHLIch 1967).

Die Zusammenhänge sind komplizierter als ursprünglich angenommen. Zunächst wäre zu vermuten gewesen, daß bei der Abdrift der limnischen Plankter in die Halinitätsbereiche - und vice versa der marinen Formen in brackige Zonen - das Absterben der Phyto- und Zooplankter gleichmäßig entsprechend dem geänderten Salzeinfluß einsetzt. Grundsätzlich sind Aestuarbereiche geänderter Halinität Mortalitätszonen für die hierher verdrifteten und nach ihrer ökologischen Valenz nicht adaptierten Arten. Diese Tatsache gilt in etwa für das Zooplankton: Nach Beobachtungen von Grere (1968) kann sich unter den Extrembedingungen des Aestuars nur im externen Aestuarbereich ein arten- und individuenreiches marines Zooplankton entfalten. Die Abnahme der Halinität - verbunden mit ungünstigen hydrographischen Bedingungen - reduziert elbaufwärts in steigendem Maße die Arten- und Individuenzahl, so daß die Limitierung des marinen Zooplanktons etwa bei $10 \% \mathrm{~S}$ erfolgt (Abb. 3). Nur vereinzelt dringen marine Plankter weiter in die Unterelbe vor, wobei das Eindringen diskontinuierlich ist: Gewöhnlich findet die radikale Abnahme mariner Formen zwischen 15 bis $10 \% \mathrm{~S}$ (Cuxhaven-Otterndorf) statt, während das limnische Zooplankton zwischen 3 bis $5 \%$ S größtenteils abstirbt. Die dazwischenliegende Flußstrecke - mit Halinitätsbereichen von 10 bis $5 \% \mathrm{~S}$ - ist als Verödungszone des Elbe-Aestuars für marine und limnische Plankter anzusehen (GIERE 1968).

In verstärktem Umfang macht sich beim Phytoplankton ein spezifisches AestuarPhänomen geltend, nämlich die Tatsache, daß durch das Auf- und Abpendeln der Tidenströmung die Verweilzeit der Arten ihre individuelle Lebenszeit übertriff mit der Folge, daß ihr Absterben bereits in der Gedeihzone vor sich geht (vgl. Abb. 4 unten). Abdriftend aus dieser Zone ist dann im wesentlichen nur noch Detritus festzustellen, sofern dieser nicht ebenfalls noch vorher bakteriell abgebaut worden ist. Eine Detritus-Verdriftung kommt ferner nur aus den limnischen Populationen in Frage, während der thalassogene Detritus aus strömungstechnischen Gründen (bei größerer räumlicher Verteilung) nicht sekundär mit dem Flutstrom in die oberen Brackwasserzonen verfrachtet wird (dagegen können Feinsände, Diatomeen-- und Foraminiferenschalen sehr weit aufwärts gelangen). Aus diesen Zusammenhängen resultiert eine Ansammlung von limnogenem Detritus in dem mixo-oligohalinen Gebiet des Aestuars. (Uber die Intensität der Dekomposition dieses Detritus laufen zur Zeit Untersuchungen.) Die Absterbe- und Abbauprozesse des limnischen Phytoplanktons haben zur Folge, daß der entstehende Detritus, vermischt mit anorganischen, durch die Turbulenz schwebend gehaltenen Stoffen (vornehmlich Feinsande und Tonmineralien) eine für ein solches Aestuar charakteristische "Trübungszone" ausbildet, deren jeweilige Lage von der Wasserführung und den speziellen Tideverhältnissen abhängt (Abb. 4 oben). Grundlage dafür ist die Erstreckung der mittleren Flut- und Ebbewege, die ein Wasserteilchen zurücklegt: Hierfür hat HENSEN (1953) ein Berechnungsverfahren angegeben und festgestellt, daß im Brackwassergebiet die mittlere Flutstromdauer am Gewässergrund größer ist als die mittlere Ebbstromdauer, während an der Oberfäche die Verhältnisse 
Einfluß der Elbe auf die Verunreinigung der Nordsee

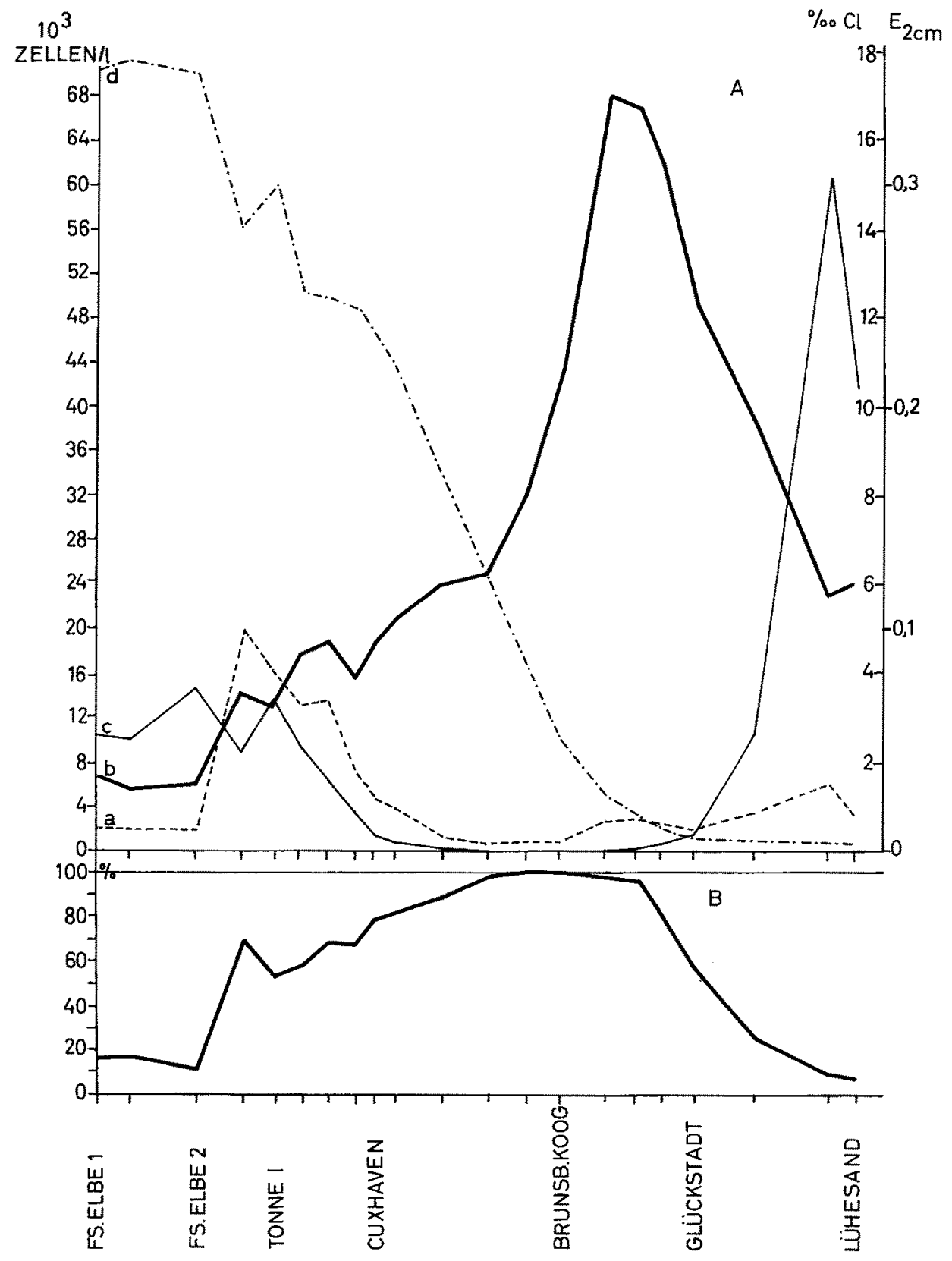

Abb. 2: Verteilung von Phytoplankton, Trübung und Salzgehalt im Elbe-Aestuar. A: Phytoplankton (tot) $=$ Kurve a; Trübung (Extinktion) $=$ Kurve b; Phytoplankton (lebend) $=$ Kurve c; Halinitat in $\% \mathrm{Cl}=$ Kurve d. B. Prozentualer Anteil der Diatomeenschalen an der Gesamtzellenzahl. (Nach NöTHLrCr 1967) 
umgekehrt sind (vgl. Luchr 1964). Dies hat zur Folge, daß an der Flußsohle das Sediment vorwiegend flußaufwärts verfrachtet wird (vgl. Simon 1953), an der Oberfläche dagegen elbabwärts. Dabei gibt es im Aestuar einen Abschnitt, an dem die beiden Gezeitenströme im Gleichgewicht zueinander stehen. Dieser „kritische Gleichgewichtspunkt" (HENSEN 1953) ist besonders wichtig, denn oberhalb von ihm überwiegt die

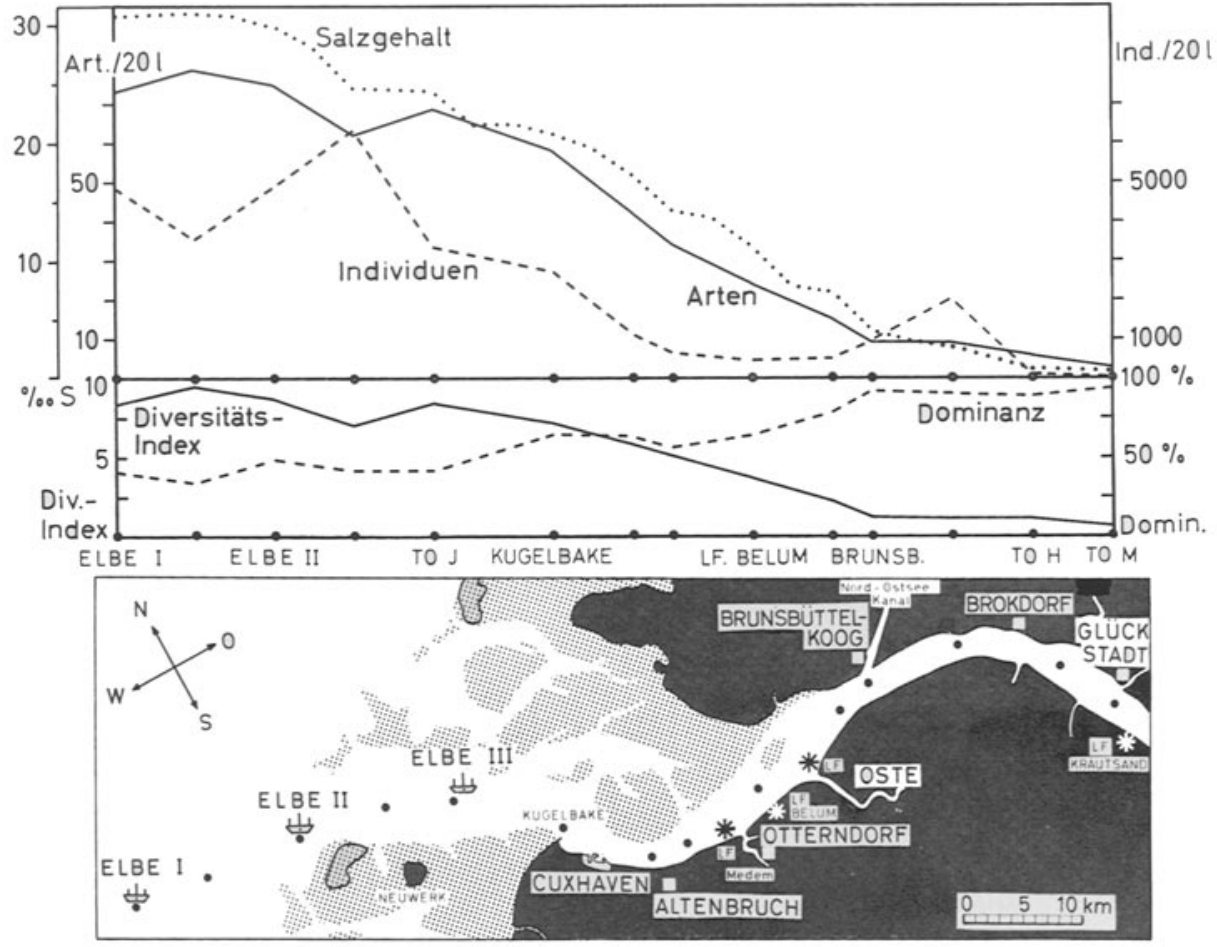

Abb. 3: Artenmannigfaltigkeit und Individuenzahl des marinen Zooplanktons im Elbe-Aestuar. (Nach GIERE 1968)

stromab- und unterhalb von ihm die stromaufgerichtete Sediment- und Seston-Wanderung. Die Folge ist, daß hier eine Anhäufung von Seston-Material sowohl limnischen als auch marinen Ursprungs stattfindet, zumal Flut- und Ebbstrom hier in unmittelbarem Gegensatz zueinander treten und die stärksten Mischungsvorgänge bedingen (LUCHT 1964). Die dadurch hervorgerufenen Turbulenzen beschleunigen den vertikalen Austausch von Wassermassen, so daß immer wieder neben Feinsand auch Diatomeenschalen und Grobdetritus in obere Wasserschichten transportiert werden. Die aufgewirbelten Trübstoffe tragen wesentlich zum Aufbau der Trübungszone bei, sind aber zunächst nur als sichtbares Zeichen des hydrographischen Geschehens zu werten. Die Lage der Zone im Aestuar wird durch die periodischen und unperiodischen Schwankungen des Gezeitenstromes und ferner durch die unterschiedliche Oberwasserführung bestimmt. Obwohl daraus eine variable räumliche Trübungsverteilung während eines Jahres- 
zyklus resultiert, befindet sich das Maximum stets im $\beta$-mesohalinen bis oligohalinen Bereich (Abb. 4 oben). Postma \& Kalle (1955) bezeichnen den Vorgang in dieser Zone als „Sinkstoff-Falle" (vgl. auch Koske et al. 1966).
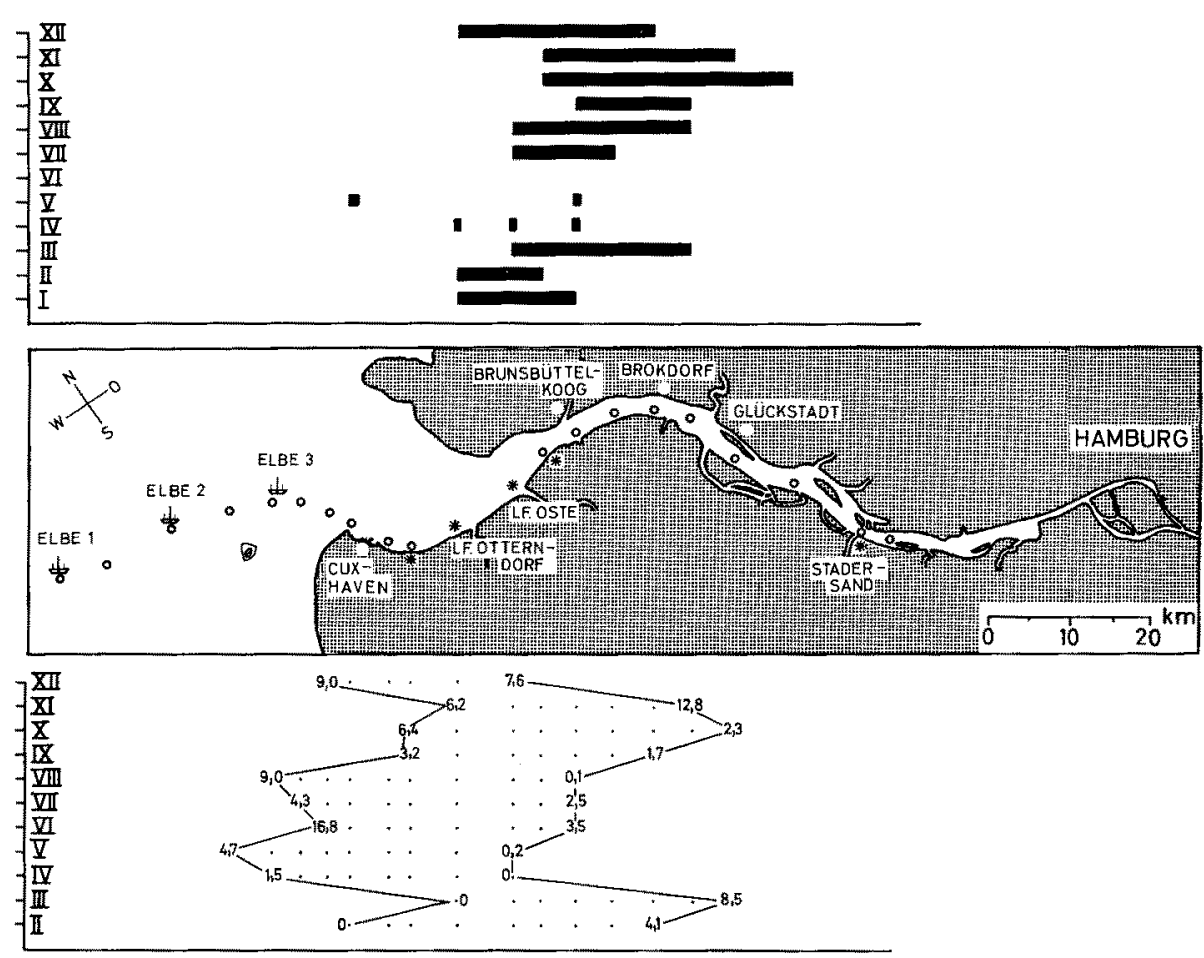

Abb. 4: Phytoplankton und Trübungszone im Elbe-Aestuar (1965). Oben: Jahreszeitliche Verlagerung der Trübungszone. Unten: Vorkommen mariner und limnischer Phytoplankter an der unteren und oberen Grenze des Meso-Oligohalinikums (gepunktet). Die Zahlenwerte geben den Anteil der an der Grenze noch lebend angetroffenen Phytoplankter an (in \% zum Populationsmaximum). (Nach NöTHLхсн 1967)

Es sei angefügt, daß auch im Bereich der Außenelbe - zwischen den Feuerschiffen Elbe II und III - eine Trübungszone, wenn auch geringer Intensität, vorhanden ist, die ebenfalls hydrographisch bedingt ist, allerdings weniger Detritus als Feinsände und Tonmineralien enthält.

Mit diesen Unterlagen kann die "Schmutzführung" der Elbe diskutiert werden. Hiermit im Zusammenhang steht die wichtige Frage nach der biologischen Selbstreinigungspotenz und damit nach der „Belastbarkeit“ eines Aestuars. Wie dargestellt, haben wir in einem Aestuar zunächst eine natürliche „Verschmutzung“ durch die auf das Absterben der produzierten Biomasse zurückgehende Detritus-Anreicherung. Produktion und Sterberate stehen dabei in Relation, d. h. die Menge des anfallenden Detritus wird neben der hydrographischen Akkumulation durch die - jahreszeitlich schwankende organische Produktion bestimmt. Eine Vermehrung dieser Produktion durch anthropo- 
gene Einleitung von organischen Abwässern oder Planktonnährstoffen wird dieses Gleichgewicht beeinflussen, in seiner Struktur jedoch nicht grundsätzlich ändern. Für die Erfassung dieser Relationen ist eine stoffwechseldynamische Bilanzierung erforderlich, die bei den komplexen hydrographischen Aestuarbedingungen allerdings schwierig ist; Ansätze hierzu werden zur Zeit in der Hydrobiologischen Abteilung des Hamburger Zoologischen Staatsinstituts erarbeitet. Eine solche Analyse der Kreislaufverhältnisse in den einzelnen Aestuarzonen (Nahrungsketten, Relation von Assimilation zu Dissimilation, Freßraten des Zooplanktons) wäre die Voraussetzung für eine Erfassung der stoffwechseldynamischen Situation. Eine Quantifizierung dieser Kreislaufprozesse wird es dann ermöglichen, für die verschiedenen hydrographischen Bereiche die Selbtsreinigungspotenzen der Biocoenosen zu bewerten. Damit wäre dann auch die Unterlage gegeben für eine Aussage über die Auswirkungen zusätzlicher Abwassereinflïsse, seien sie positiver oder negativer Art. Es kann von der Annahme ausgegangen werden, daß ein Aestuarsystem durch die natürlichen Gegebenheiten sozusagen auf eine gesteigerte Dekompositionsleistung eingestellt ist, die Selbstreinigungspotenzen also um ein Vielfaches die eines normalen Flußvorfluters übersteigen. Entscheidend für die Verarbeitung erhöhter organischer Einleitungen, also z. B. häuslicher Abwässer, ist die Erhaltung der normalen biocoenotischen Reaktion. Giftige Abwässer würden hier zu eventuell größeren Störungen als in anderen Vorflutern führen. Andererseits bewirkt die Turbulenz der Tideströmung einen gesteigerten atmosphärischen SauerstoffEintrag, der die Aufnahmekapazität der Biocoenosen fördert.

Ein für die Frage der Selbstreinigungspotenz des Aestuars wichtiger Umstand ist in dem Vorhandensein der Trübungszone gegeben. Sie dürfte als eine Art Filter für Bakterien und Nährstoffe wirken, sozusagen als eine vom Fluß selbst geschaffene mechanische „Kläranlage“. Allerdings ist die Dekompositionsrolle noch ungeklärt (auch hierüber laufen derzeitig Untersuchungen in der Hydrobiologischen Abteilung).

Als Fazit der stoffwechseldynamischen Kreisläufe in der regionalen Kettenfolge der Flußmündung ergibt sich ein Abstrom von gelösten Nährstoffen in den „Vorfluter Nordsee“. Dieser Eintrag erhöht das trophische Niveau zumindest der Küstenbereiche. Hierauf basiert die reiche Bodenfauna der sandig-schlickigen Sedimentzonen der polyhalinen und weiter der vorgelagerten euhalinen Bereiche (vgl. CASPERs 1948). Ferner erfahren die der Außenelbe anliegenden Wattenzonen eine gesteigerte organische „Düngung“. Hier macht sich der Prozeß der sekundären „Verschmutzung“, also die erhöhte Phytoplankton-Produktion auf Grund des Einstromes gelöster Nährstoffe, geltend. Die Watten stellen Auffangzonen für das Seston (Bioseston + mineralische Bestandteile $=$ Abioseston) dar; sie sind in ihrer biocoenotischen Struktur auf die Aufarbeitung solcher organischen Zufuhr eingestellt. Somit bietet sich die Hypothese an, daß gerade hier eine vom Menschen gesteigerte Zufuhr von organischem Restmaterial zur Dekomposition kommen könnte ohne wesentliche strukturell-biocoenotische Beeinträchtigung. Natürlich ist nicht "das Watt" als geographische und biocoenotische Einheit aufzufassen. Zum weiteren muß eine Nutzung des Wattes - z. B. für Muschelkulturen oder seine Bedeutung als Laichgebiet für Fische, Krebse etc. berücksichtigt werden. Es sollte nur als Gesichtspunkt darauf hingewiesen werden, daß im Flachkïstenbereich des Meeres potentielle Abwasser-Auffanggebiete sozusagen von der Natur "vorbereitet" sind, die bei einer erforderlichen Zuleitung von menschlichem Abwasser besser als Vor- 
fluter-Regionen fungieren könnten als andere, geringer trophische Meereszonen. Es handelt sich um Hypothesen, deren Bestätigung umfangreiche Untersuchungen - am $\mathrm{Ob}$ jekt und im Experiment - erfordert.

Rückblickend sei herausgestellt: Flußmündungen mit Aestuarcharakter stellen Zonen natürlicher Ansammlungen von Detritus dar. Dieser wird zum großen Teil bereits im Aestuar selbst zur Dekomposition geführt; Trübungszonen fungieren als SinkstoffFallen. Die in den "Vorfluter Meer" einströmenden Nährstoffe provozieren eine „Sekundärverschmutzung ", deren Restbestandteile in erheblichem Umfang in den Wattenbereichen abgelagert und weiter abgebaut werden. Eine Steigerung dieser stoffwechseldynamischen Kreislaufprozesse durch Abwassereinleitungen ist in gewissem Umfang möglich, da der zusätzliche Detritus-Anfall in einem bereits auf den Uberschuß der De-kompositionsprozesse „eingestellten“ Equilibrium aufgefangen und an Orten deponiert wird, die durch die geographischen und hydrographischen Gegebenheiten hierfür praem disponiert sind. Es wird Aufgabe der marinen Abwasserbiologie sein, die Grenzen der Dekompositionspotenz solcher Vorfluterbereiche festzustellen.

\section{ZUSAMMENFASSUNG}

1. Der Zustrom von organischem Restmaterial aus den Flüssen in das Meer stellt eine Art von „Verschmutzung" dar mit der Folge einer Erhöhung des Trophiespiegels. Der Umfang der Verdünnung im „Vorfluter" und allgemein die geographischen und hydrographischen Situationen bestimmen das aus dem Angebot an Nährstoffen resultierende marine Equilibrium.

2. Im Elbe-Aestuar haben wir alle Ubbergänge zwischen mixo-oligohalinen und -polyhalinen Zonen, deren Lage in den Jahreszeiten und zum Teil kurzfristig stark verschoben werden kann. Dadurch ergeben sich für das Benthos extrem astatische Verhältnisse.

3. Durch den Tidewechsel wird das Plankton in den einzelnen Halinitätsbereichen länger zurückgehalten als in einem einseitig abfließenden Strom. Entsprechend dieser verlängerten Verweilzeit können sich typische Plankton-Biocoenosen in regionaler Sukzession ausbilden. Das Phytoplankton mit seiner schnellen Reproduktionsrate ist hierbei besonders begünstigt.

4. Aus hydrographischen Gründen entsteht im oligo- bis mesohalinen Bereich eine Trübungszone, deren Material vornehmlich aus den Resten der limnischen Produktion (und Tonmineralien) besteht. Durch die natürlichen Gegebenheiten ist ein Aestuar-System sozusagen auf eine gesteigerte Dekompositionsleistung eingestellt, so $\mathrm{daß}$ eine anthropogene Zugabe von organischem Restmaterial ohne stärkere Beeinträchtigung des biocoenotischen Systems möglich ist.

5. Im Vormündungsgebiet der Elbe bewirkt der Einstrom großer Mengen gelöster Nährstoffe aus dem Aestuar eine Erhöhung des trophischen Niveaus und eine "Sekundärverschmutzung“, deren Auffangzonen die tieferen Meeresflächen und die Wattenbereiche darstellen. Die Watten sind biocoenotisch auf die Aufnahme großer Mengen an Detritusmaterial eingestellt; es wird die Frage diskutiert, wieweit in solche Bereiche auch eine zusätzliche Einleitung von organischem Abwasser ohne wesentliche Beeinträchtigung des biologischen Equilibriums möglich ist. 


\section{ZITIERTE LITERATUR}

Bursche, E.-M., KühL, H. \& ManN, H., 1958. Hydrochemie und Phytoplankton in der Unterelbe. Veröff. Inst. Meeresforsch. Bremerh. 5, 165-192.

CASPERS: H., 1949. Okologische Untersuchungen über die Wattentierwelt im Elbe-Aestuar. Zool. Anz. (Suppl. Bd) 13, 350-359.

- 1952. Bodengreiferuntersuchungen über die Tierwelt in der Fahrrinne der Unterelbe und im Vormündungsgebiet der Nordsee. Zool. Anz. (Suppl. Bd) 16, 404-418.

- 1954. Biologische Untersuchungen über die Lebensräume der Unterelbe und des Vormündungsgebietes der Nordsee. Mitt. geol. StInst. Hamb. 23, 78-85.

- 1955. Limnologie des Elbe-Aestuars. Verh. int. Verein. theor. angew. Limnol. 12, 613-619.

- 1958. Biologie der Brackwasser-Zonen des Elbe-Aestuars. Verh. int. Verein. theor. angew. Limnol. 13, 687-689.

- 1959a. Die Einteilung der Brackwasser-Regionen in einem Aestuar. Archo Oceanogr. Limnol. 11 (Suppl.), 155-169.

- 1959b. Vorschläge einer Brackwassernomenklatur. Int. Revue ges. Hydrobiol. 44, 313-316.

- 1961. Biologie des Elbe-Aestuars. Vorwort zu "Elbe-Aestuar I“. Arch. Hydrobiol. Suppl. $B d 26,1-4$.

CASPERS, H. \& KARBE, L., 1967. Vorschläge für eine saprobiologische Typisierung der Gewässer. Int. Revue ges. Hydrobiol. 52, 145-162.

DAfL, F., 1892. Untersuchungen über die Tierwelt der Unterelbe, 6. Ber. Comm. wiss, Unters. dt. Meere Jg. 21 (3), 149-185.

GIERE, O., 1968. Die Fluktuationen des marinen Zooplanktons im Elbe-Aestuar. Beziehungen zwischen Populationsschwankungen und hydrographischen Faktoren im Brackwasser. Arch. Hydrobiol. (Suppl. Bd „Elbe-Aestuar III") (im Druck).

Grimm, R., 1968. Biologie der gestauten Elbe. Die Auswirkungen der Staustufe Geesthacht auf die benthale Fauna im oberen Grenzbereich des Elbe-Aestuars. Arch. Hydrobiol. (Suppl.Bd "Elbe-Aestuar III") (im Druck).

Hamburgische Elb-Untersuchung. T. 1-9. 1903-1909. In: Jahrbuch der Hamburgischen Wissenschaftlichen Anstalten. Beih. 2 (= Mitt naturh. Mus. Hamb.) 19, 65-210. 1902. 1903 - 26, 249-279. 1908. 1909.

Hensen, W., 1953. Das Eindringen von Salzwasser in die Gezeitenflüsse und ihre Nebenflüsse, in Seekanäle und Häfen. Mitt. bannover. VersAnst. Grundb. WassBau 3, 20-50.

Kirchenpauer, J. U., 1862. Die Seetonnen der Elbmündung. Abh. Geb. Naturw. Hamburg 4, $1-59$.

Koske, P. H., Krumm, H., Rheinheimer, G. \& Szekielda, K. H., 1966. Untersuchungen über die Einwirkung der Tide auf Salzgehalt, Schwebstoffgehalt, Sedimentation und Bakteriengehalt in der Unterelbe. Kieler Meeresforsch. 22, 47-63.

KотнÉ, P., 1961. Hydrobiologie der Oberelbe. Natürliche, industrielle und wasserwirtschaftliche Faktoren in ihrer Auswirkung auf das Benthos des Stromgebietes oberhalb Hamburgs. Arch. Hydrobiol. Suppl. Bd 26 („Elbe-Aestuar I“), 221-343.

KüH, H., 1966. Der Abfluß der Elbe im Jahre 1965 und seine Wirkung auf Salzgehalt, Plankton und Bewuchsbildung bei Cuxhaven. Veröff. Inst. Meeresforsch. Bremerh. 10, 61-70.

KüHL, H. \& ManN, H., 1953. Beiträge zur Hydrochemie der Unterelbe. Veröff. Inst. Meeresforsch. Bremerh. 2, 236-268.

Lucht, F., 1953. Die Sandwanderung im unteren Tidegebiet der Elbe. Dt. bydrogr. Z. 6, 186-207.

- 1964. Hydrographie des Elbe-Aestuars. Arch. Hydrobiol. Suppl. Bd 29 („Elbe-Aestuar II“), $1-96$.

Nöтнцсн, I., 1967. Untersuchungen über den Schlickhaushalt in der Unterelbe mit besonderer Berüicksichtigung der biologischen Komponenten. Mitt. Wass,- u. Schiffabrtsdir. Hamb. 17 $(1 / 2), 1-155$.

Postma, H. \& Kalde, K., 1955. Die Entstehung von Trübungszonen im Unterlauf von Flüssen, speziell im Hinblick auf die Verhältnisse in der Unterelbe. Dt. bydrogr. Z. 8, 137-144. 
Schulz, H., 1961. Qualitative und quantitative Plankton-Untersuchungen im Elbe-Aestuar. Arch. Hydrobiol. Suppl. Bd 26 („Elbe Aestuar I“), 5-105.

Simon, W. G., 1953. Untersuchungsergebnisse an Grundproben aus dem Gebiet der Elbe zwischen Scheelenkuhlen und Cuxhaven und ihre Ausdeutung hinsichtlich der Sandwanderung 1951. Mitt. Wass.- u. Schiffabrtsdir. Hamb. 8, 1-153.

\section{Diskussion im Anschluß an den Vortrag CASpers}

KorRINGA: The Waddensea mud flats are rich in animal life in the surface layer. Young fish and shrimp migrate with the flood to these feeding grounds. Excess of organic matter leads to formation of soft black mud with a considerably poor variety of food organisms for fish and shrimp. This will reduce the value of such mud flats as feeding grounds, to denaturation of the nursery grounds. Too much organic matter in this zone of natural decomposition may easily lead to reduction of oxygen in the water layers close to the bottom. Decomposition by microorganisms requires much oxygen. Mussels can deposit so much organic material under themselves causing a lowering of the oxygen level. A mass mortality of Coscinodiscus demonstrated in the Dutch Waddensea that a renewed catastrophe is possible through oxygen depletion.

Postma: I would like very much to support the warning of Dr. KorringA that the Waddensea may not be able to receive considerably more organic matter than it already does at present. Observations in the yet unpolluted western Waddensea show that a significant "natural" oxygen undersaturation occurs there regularly. I do not believe that many parts of this area support a much greater load of organic materials without harmful consequences. No increase of this load should take place without extensive research. In the second place I would like to ask Dr. CAspers whether he has observations about the particle size in the turbidity zones; since these zones select a specific size range (particles with a specific settling velocity), they would also accumulate a specific range of sewage particles.

KoRRINGA: The Dutch Waddensea is very important for mussel farming. The Delta project will eliminate the mussel farming in Zealand. Therefore efforts will be made to raise the mussel production in the Waddensea. Scientific research is in progress on this subject. There are already now indications that oxygen content of the water in the bottom layers could be the limiting factor. Sewage brought into the Waddensea requires a lot of oxygen for its biodegradation. Every quantity of sewage could mean a lower production level of mussels. WC prefer mussels above sewage in the Waddensea.

KöNIG: Eine Bemerkung zur Frage des Vortragenden, ob man die Abbauzonen im Watt zur Aufnahme anthropogener Abfälle benutzen könne: In Schleswig-Holstein haben wir in einigen besonderen Fällen gewagt, sie mit "Ja“ zu beantworten. Während im allgemeinen bei der Abwasserreinigung mechanische und biologische Behandlung gefordert werden, haben wir uns mit der mechanischen Klärung dort begnügt, wo das $\mathrm{Abwasser}$ von Inselgemeinden in ausgesprochene Schlickwatten geleitet wird, wo (1) ohnehin solche Abbauvorgänge natürlicherweise im polysaw proben Bereich erfolgen, (2) Menschen normalerweise nicht hingehen und (3) genügend weite Entfernung vom Badestrand gegeben ist (Beispiele: Norddorf und Nebel auf Amrum, Wyk auf Föhr). An diesen Stellen sind, da es sich um ausgesprochene Badeorte handelt, toxische Stoffe in wesentlichen Mengen im Abwasser nicht vorhanden. Vor den Kanten unserer Inseln im Wattenmeer liegen fast stets mehr oder weniger breite Faulschlickzonen, wo die Massen von Algen und Seegras zusammengetrieben werden und dort verfaulen.

Caspers: Id danke Ihnen, Herr Dr. König, für diesen Hinweis. Das sind die Bereiche, an die ich gedacht hatte, als ich meine hypothetische Frage stellte, also nicht etwa die Zonen der Muschelkulturen, die Herr Professor Korringa hier verteidigte. Es gibt natürliche Faulbereiche am Meeresstrand, die eventuell in ihrer Dekompositionsleistung noch gesteigert werden können, ohne daß sonst ein biologischer Schaden angerichtet wird. An solche Zonen kann man denken, wenn man nun schon $\mathrm{Abwasser}$ einleiten muß. Natürlich wäre es am besten, wenn $A b w a s s e r-$ 
einleitungen in das Meer überhaupt unterbleiben könnten (auch mir sind im Wattenbereich Muscheln lieber als Abwasser!), aber es geht ja um die Frage, wohin notwendige, unvermeidliche Abwassereinleitungen gegeben werden sollten. Es wäre Aufgabe der Biologen, audh die Industrie auf mögliche Einleirungsorte hinzuweisen und andererseits darauf zu dringen, daß andere Küstenbereiche, die zu wertvoll sind, als daß wir sie verderben lassen, verschont bleiben.

LÜNeBURG: Können Sie die Auffassung bestätigen, daß die enormen Trübungsspitzen im ElbeAestuar mit einigen Gramm Trockensubstanz pro Liter Substanzen sind, die enorm absorptiv auf alle möglichen Chemikalien wirken? Diese kommen doch nur in den Marsch-Aestuaren vor (wie z. B. Ems, Schelde, Weser, Elbe). Die Trübungswolke ist zum großen Teil eine Funktion der anstehenden Schlick-, also Silt-Sedimente auf beiden Seiten und an der Sohle des Flusses. Wenn Sie die Trübungswolke in Ufernähe verfolgen, so ist diese mit einer Körnung von durchschnittlich $20 \mu$ weit hinaus in den polyhalinen und anderseits in den limnischen Bereich zu verfolgen. Die Trübungswolke ist doch in erster Linie eine Funktion der anstehenden Sedimente.

CASPERS: Wir haben sehr genaue Messungen über die Partikelgröße der einzelnen Komponenten, also der Tontrübe und des organischen Detritus; weiter ist natürlich auch ein starker Anteil an Quarzkörnern vorhanden, deren Transport im freien Wasser von der Stärke der Strömung abhängig ist. Ich hatte gezeigt, daß unterhalb des Schwellwertes bei Stauwasser die Grobbestandteile sedimentieren und erst bei starkem Flut- und Ebbstrom wieder aufgeschwemmt werden, während die Tontrübe sich stärker im freien Wasser hält. Nur wird diese Tontrübe nicht gleich abgeführt: sie bleibt in den auf- und abpendelnden Wasserkörpern, so daß also auch hier eine Stetigkeit der Verhältnisse - abgesehen von dem Wechsel der Großlage der einzelnen Halinitätszonen - gegeben ist. Die Zusammensetzung der Wassertrübe wechselt also in den einzelnen Tidephasen sehr erheblich.

Neumann: Zu den Strömungsverhältnissen: Bei Glückstadt liegt die Stelle, bei der Flut- und Ebbstrom von oben bis unten gleichzeitig eintreten. Unterhalb haben wir ein Gebiet - vor allem bei Cuxhaven - in dem der Flutstrom am Boden erheblich früher eintritt als an der Oberfläche, so daß der Strom an der Oberfläche noch ausläuft, während er am Boden schon einläuft. Es bestehen ferner erhebliche Unterschiede in den Stromgeschwindigkeiten und Eintrittszeiten, wenn man die rechte und linke Seite des Flusses unterhalb Brunsbüttelkoog betrachtet. Die Stelle, an der Flut- und Ebbstrom zwischen Feuerschiff Elbe 3 und Elbe 2 von oben bis unten gleichzeitig eintritt, ist mir nicht bekannt.

CASPERS: Ich habe natürlich in dieser zusammenfassenden Darstellung die ganze Mikrostruktur in den einzelnen Zonen nicht behandeln können; ich mußte versuchen, sozusagen "reine Linien" herauszuarbeiten, statt auf die Einzelheiten zu kommen. Gerade in den von Ihnen erwähnten Verhältnissen in der Außenelbe haben wir ein sehr kompliziertes hydrographisches System, wie es sich auch in der Verteilung des lebenden Planktons spiegelt. Wenn man mit dem Schiff fährt, kann man häufig beobachten, daß eine Stromkante vorhanden ist, die zum Teil quer zum Strom geht, wo also Wasser über das Wattengebiet eindringt in das ab-oder zufließende Wasser des Aestuar. Und auch im Bereich des Aestuar-Schlauches haben wir zum Teil erhebliche Unterschiede zwischen oben und unten und zwischen links und rechts. Aber ich glaube, daß es im Rahmen eines solchen Referates nicht möglich wäre, auf diese Komplikationen noch näher einzugehen, zumal die Fragen der trophischen Gesamtstruktur im Vordergrund zu stehen hatten. 